\title{
Scientific papers on musculoskeletal radiology presented at ECR 2020
}

\author{
Anagha P Parkar ${ }^{1,2}$ (D)
}

Received: 2 March 2020 / Accepted: 5 May 2020 / Published online: 16 May 2020

(C) European Society of Radiology 2020

The number and rate of musculoskeletal examinations performed at an institution vary depending on where or which type of hospital or institution one works at. Musculoskeletal radiology makes up a large portion of all imaging, as reflected in the number of abstracts and scientific sessions at this years' ECR. Nine scientific sessions with musculoskeletal imaging abstracts will be presented in the upcoming ECR.

As in previous years, the knee and lower extremities are the largest areas of research, comprising a third of the sessions, and only one session on upper extremities. However, there is a clear shift in the areas of research. In previous years, the MRI was the main modality of research. This year, many abstracts include US, reflecting the general shift in radiology practice where musculoskeletal radiologists are more "hands on" and actively involved in patient communication and treatment.

The cutting-edge research is the emerging field of artificial intelligence (AI), which includes deep learning and machine learning. AI is moving steadily from "techno-optimism" towards "techno-reality". One interesting paper investigated the efficacy of deep learning to differentiate between benign and malignant soft tissue masses in US [1]. US is often considered not as reliable as other modalities, due to inter-operator variability. The capability of AI assisting in US and reducing this variability holds great promise for the future.

Further presentations focus on AIs improvement in assessing femoroacetabular impingement on MRI, detection of pediatric tibial fractures, bone age assessment and detection of anterior cruciate ligament ruptures. The ability of AI to aid and improve a radiologists performance should not be considered a threat to radiology, but rather an assistant, as workloads

Anagha P Parkar

apparkar@gmail.com

1 Department of Radiology, Haraldsplass Deaconess Hospital, Ulriksdal 8, 5009 Bergen, Norway

2 Department of Clinical Medicine, Faculty of Medicine and Dentistry, University of Bergen, Bergen, Norway do not seem to decrease and complexity of examinations and radiology reports are ever increasing.

An area which has been around for some time, but has not been extensively implemented in daily practice yet, is dualenergy CT in musculoskeletal applications [2]. Gout assessment is well known, however, the other possibilities such as perfusion quantification, lag, perhaps because the clinical usefulness of such an evaluation is not there yet. The gold standard for assessing bone marrow edema (BME) is by far MRI. Dual-energy CT assessment of BME shows promise in aiding insufficiency fracture diagnosis, where it is may be notoriously difficult to depict actual fracture lines on CT. However, as another research paper showed that BME on dual-energy CT was also observed in non-traumatic hip pain cases, the same can be observed on dual-energy CT images in cases without trauma, the usefulness depicting BME in subtle fractures to differentiate fractures from non-traumatic BME, is yet to be resolved [3].

The new kid on the block is sarcopenia [4]. This theme is a very important field, which has long been neglected. Sarcopenia is defined as low muscle mass and either low muscle strength and/or low physical performance. It is mainly a condition in the elderly, but can also occur in patients with developmental or lifestyle diseases. As muscles and physical activity have a beneficial effect on several other organs, such as brain, cardiovascular as well as liver, to mention a few, the loss of muscle negatively affects the whole patient. The level of sarcopenia in a patient can predict the outcome of surgical treatment. As such, in the future radiologists will have to learn to assess and report on muscle mass and body composition in general.

The abstracts of musculoskeletal scientific presentations at this years' ECR are varied and cover a wide range of fields. Still, one field is not sufficiently represented: 4D-CT [5]. Dynamic CT imaging is a very exciting area, which when performed correctly, yields images with great anatomic detail as well as function. 4D-CT will for sure become a standard for all complicated fractures and joint pathology in the future, 
especially the possibility of combining with 3D-printing, will strongly influence orthopedic treatment and surgery in the years to come.

Funding information The authors state that this work has not received any funding.

\section{Compliance with ethical standards}

Guarantor The scientific guarantor of this publication is Anagha P. Parkar

Conflict of interest The authors of this manuscript declare no relationships with any companies, whose products or services may be related to the subject matter of the article.

Statistics and biometry No complex statistical methods were necessary for this paper.

Informed consent Written informed consent was not required for this paper, as it is editorial.

Ethical approval Institutional Review Board approval was not required because it is an editorial.
Methodology $•$ No specification required, as it is an editorial

\section{References}

1. RPS 1010a - Artificial intelligence (AI) and new techniques in MRI. ECR 2020 Book of Abstracts. Insights Imaging. https://doi.org/10. 1186/s13244-020-00851-0

2. Rajiah P, Sundaram M, Subhas N (2019) Dual-energy CT in musculoskeletal imaging: what is the role beyond gout? AJR Am J Roentgenol 213:493-505

3. RPS 110 - Cartilage, bone marrow oedema, tissue and body imaging. ECR 2020 Book of Abstracts. Insights Imaging. https://doi.org/10. 1186/s13244-020-00851-0

4. Lee K, Shin Y, Huh J et al (2019) Recent issues on body composition imaging for sarcopenia evaluation. Korean J Radiol 2:205-217

5. Gondim Teixeira PA, Formery AS, Hossu G et al (2017) Evidencebased recommendations for musculoskeletal kinematic 4D-CT studies using wide area-detector scanners: a phantom study with cadaveric correlation. Eur Radiol 27:437-446

Publisher's note Springer Nature remains neutral with regard to jurisdictional claims in published maps and institutional affiliations. 